\title{
J ust another Sebaceous Cyst?? - An Epidermoid Cyst of Head \& Neck Region: A Case Report
}

\author{
Boricha V*, Shetty N, Vachhani D, Bangera D and \\ Surabhi V \\ Department of Oral \& Maxillo-Facial Surgery, AJ \\ Institute of Dental Sciences, India \\ *Correspondling author: Vishal Kumar Boricha, \\ Department of Oral \& Maxillo-Facial Surgery, AJ \\ Institute of Dental Sciences, Karnataka, India
}

Received: February 05, 2018; Accepted: March 21, 2018; Published: April 24, 2018

\begin{abstract}
Epidermoid cysts are developmental, benign, cutaneous cysts which rarely occurs in head and neck region. They constitute 1.6 to $6.9 \%$ of all cysts in the head and neck area. Here we report a case of 34 year old male patient with two swellings on his right neck region. Ultrasonography of the lesion was suggestive of sebaceous cyst. Complete excision of lesion was carried out and sent for histopathological examination. On the basis of clinical and histopathological features, a final diagnosis was given as an epidermoid cyst for both the swellings.
\end{abstract}

Keywords: Epidermoid cyst; Head \& Neck region; Ultrasonography

\section{Introduction}

Epidermoid cysts are developmental, benign, cutaneous cysts which are commonly found on scalp followed by trunk [1]. These cysts are the benign cystic malformations that are derived from the ectoderm.

They rarely occur in neck region and constitute 1.6 to $6.9 \%$ of all cysts in the head and neck area. They represent less than $0.01 \%$ of all cysts of the oral cavity $[1,2]$.

An epidermal cyst is derived from epidermis, and is formed by cystic enclosure of epithelium within the dermis that becomes filled with keratin and lipid-rich debris [1]. They are slow growing lesions and remain asymptomatic until or unless secondarily infected.

Diagnosis is mostly by clinical examination and by ultrasonography which is frequently used in initial stages to confirm the cystic nature of the lesion $[3,4]$. Excision biopsy is carried out followed by the confirmatory diagnosis.

Treatment for these lesions is surgical excision of the cyst (Nicollas et al.; Golden \& Zide). Recurrence after surgery is rare. A malignant evolution has only been seen in the teratoid type and was reported to have an incidence of $0.5 \%$ [2].

\section{Case Presentation}

A 34 year old male reported to the department with two swellings on the right side of the neck. Both the swellings were present since 12 years. The swellings were asymptomatic and insidious in onset. There was no history of trauma or any discharge. On examination the first swelling was present inferiorly to the lower border of the mandible measuring $15 \times 20 \mathrm{~mm}$. Second swelling was present on the right lateral side of neck; anterior to the sternocleidomastoid muscle measuring 20x20 mm (Figure 1).

On palpation, the swellings were soft, non-tender, and fluctuant. The swellings were not adherent to the skin. Ultrasonography was done and it was suggestive of sebaceous cyst/epidermoid inclusion cyst for both the swellings. Complete excision of the lesions was done and it was sent for histopathological examination. Final diagnosis was given as epidermoid cyst based on clinical and histopathological
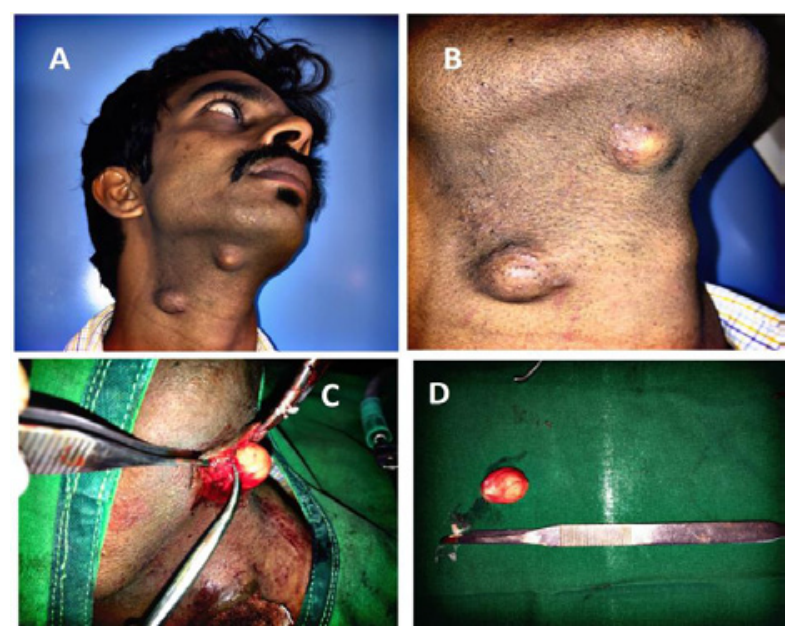

Figure 1: A. Lateral profile view of the patient.

B. Two neck swellings on the right side of the neck.

C. Intraoperative picture showing the excision of the lesion.

D. Excised specimen with the presence of punctum.

features for both the swellings.

\section{Discussion}

Dermoid cyst has been classified as true dermoid cyst, epidermoid cysts and teratoid cyst [7]. Out of all the dermoid cysts which occur in head and neck region, epidermoid cyst is the rare lesion. About $11.5 \%$ are seen in the submental region. In the neck, these cysts are usually present in the midline which gradually increase in size over the year due to accumulation of cutenous products [1].

The origin of epidermoid cyst is varied and believed to be formed by sequesteration and implantation of epidermal rest during embryonal period, occlusion of pilosebaceous glands, iatrogenic or surgical implantation of the epithelium into mesenchyme $[2,8]$.

Epidermoid cysts are slow growing and usually asymptomatic but they become inflamed or secondarily infected resulting in pain and tenderness. The cyst can occur at any age most common in the third and fourth decades of life [1]. Clinically it may present as a round
Austin J Dent - Volume 5 Issue 4 - 2018

ISSN : 2381-9189 | www.austinpublishing group.com

Boricha et al. (C) All rights are reserved
Citation: Boricha V, Shetty N, Vachhani D, Bangera D and Surabhi V. Just another Sebaceous Cyst?? - An Epidermoid Cyst of Head \& Neck Region: A Case Report. Austin J Dent. 2018; 5(4): 1114. 
firm mobile flesh colored to yellow or whitish subcutaneous nodules of variable size $[1,9]$.

Imaging has an important role in confirming the diagnosis and classifying cysts according to their relation to muscle. Ultrasound is the initial imaging modality. Epidermoid cysts are seen as welldefined cysts with multiple well defined dependent echogenic nodules within the cyst [7].

In our case the possibility of cyst originating as a result of entrapment is ruled out as there was no history of trauma and previous surgical treatment in that area.

Histopathologically, dermoid and epidermoid cysts are lined by keratinized epidermis with occasional areas of pseudostartified ciliated columnar epithelium $[1,10,11]$.

Pathological features of epidermoid cysts are oily or cheesy, tan, yellow white material and the cyst wall is a fibrous capsule usually 2-6 $\mathrm{mm}$ is thickness $[7,12]$.

Total excision is the main treatment for epidermal cystic lesions since needle aspiration or fenestration might lead to infection, pain, and complaints after treatment. Marsupalisation is another alternative for management of large cyst [7,12]. Lesions above mylohyoid muscles are operated intra-orally whereas those below the muscle are removed via an incision in the neck $[7,13,14]$.

After through clinical and radiological examination it was decided to carry out complete excision of the lesion under all aseptic measures. Local infiltration was given around the periphery of both the lesions. Eliptical incision was given. The lesion was excised and sent for histopathological examination.

On sectioning, macroscopic findings consisted of soft, cystic whitish cheesy material, probably keratin material. Histopathologic examination of $\mathrm{H}-\mathrm{E}$ stained section showed fibrovascular cystic wall lined by thin layer of stratified epithelium. A confirmatory diagnosis of epidermoid cyst was made based on clinical and histopathological features.

\section{Conclusion}

Epidermoid cysts are relatively less common in the head and neck region, hence are likely to be misdiagnosed. These cysts require thorough clinical and histopathological examination and close followup as well done to their potential for malignant transformation. Early diagnosis and removal of dermoid and epidermoid cysts are of great concern as they rarely occur in neck region.

\section{References}

1. Nicollas R, Guelfucci B, Roman S, Triglia JM. Congenital cysts and fistulas of the neck. Int J Pediatr. Otorhinolaryngol. 2000; 55: 117-124.

2. Singh CU, Bansal R, Sarkar S, Khursheed O. An Epidermoid Cyst of Head and Neck Area- A Case Report. J Adv Med Dent Scie Res. 2015; 3: 103-106.

3. Dutta M, Saha J, Biswas G, Chattopadhyay S, Sen I, Sinha R. Epidermoid Cysts in Head and Neck: Our Experiences, with Review of Literature. Indian J Otolaryngol Head Neck Surg. July 2013; 65: S14-S21.

4. Wani I, Jawaid H, Mir SR, Wani AM, Shah PS, Peerzada AH, et al. Giant epidermoid cysts. OA Case Reports. 2013; 2: 65.

5. Yasumoto M, Shibuya H, Gomi N, Kasuga T. Ultrasonographic Appearance of Derrnoid and Epidermoid Cysts in the Head and Neck. J Clin Ultrasound 1991; 19: 455-461.

6. Golden BA, Zide MF. Cutaneous cysts of the head and neck. J Oral Maxillofac Surg. 2005; 63: 1613-1619.

7. Sabhalok SS, Shetty LS, Sarve PH, Setiya SV, Bharadwaj SR. Epidermoid and dermoid cysts of the head and neck region. Plast Aesthet Res. 2016; 3 : 347-350.

8. Abrams MB, Andrews JE, Laskin DM. Epidermoid (implantation) cyst after temporomandibular joint surgery. J Oral Surg. 1977; 35: 587-589.

9. Dive AM, Khandekar S, Moharil R, Deshmukh S. Epidermoid cyst of the oute ear: A case report and review of literature. Indian J Otol. 2012; 18: 34-37.

10. Samper A, Ruiz de Erenchun R, Yeste L, Bazan A. Dermoid cyst on the auriculotemporal area. Plast Reconstr Surg. 2000; 106: 947-948.

11. Scolozzi P, Lombardi T, Jaques $B$. Congenital intracranial frontotempora dermoid cyst presenting as a cutaneous fistula. Head Neck. 2005; 27 : 429 432.

12. Mirza S, Fadl S, Napaki S, Abualruz A. Case report of complicated epidermoid cyst of the floor of the mouth: Radiology-histopathology correlation. Qatar Med J. 2014; 2014: 12-16.

13. Jham BC, Duraes GV, Jham AC, Santos CR. Epidermoid cyst of the floor of the mouth: a case report. J Can Dent Assoc. 2007; 73: 525-528.

14. Louis PJ, Hudson C, Reddi S. Lesion of floor of the mouth. J Oral Maxillofac Surg. 2002; 60: 804-807.
Austin J Dent - Volume 5 Issue 4 - 2018

ISSN : 2381-9189| www.austinpublishinggroup.com

Boricha et al. (C) All rights are reserved
Citation: Boricha V, Shetty N, Vachhani D, Bangera D and Surabhi V. Just another Sebaceous Cyst?? - An Epidermoid Cyst of Head \& Neck Region: A Case Report. Austin J Dent. 2018; 5(4): 1114. 